PROCEEDINGS OF THE

AMERICAN MATHEMATICAL SOCIETY

Volume 129, Number 5, Pages 1375-1384

S 0002-9939(00)05813-5

Article electronically published on October 20, 2000

\title{
CREATION AND PROPAGATION OF LOGARITHMIC SINGULARITIES BY INTERACTION OF TWO PIECEWISE SMOOTH PROGRESSING WAVES
}

\author{
G. LASCHON \\ (Communicated by David S. Tartakoff)
}

Dedicated to Joanna

\begin{abstract}
Our aim is to understand the non-conservation of the piecewise smooth regularity by a semi-linear interaction of two transverse progressing waves. Indeed, we know that this phenomenon occurs when the number of characteristic hypersurfaces passing through the locus of interaction, that is, a two-codimensional variety, is strictly inferior to the size of the considered first order strictly hyperbolic system. Thanks to the study of a significant example, we explain the obstruction to the piecewise smooth propagation by a loss of transmission property for the symbols describing the conormal singularities, which originates logarithmic singularities.
\end{abstract}

\section{INTRODUCTION}

We are interested in the local phenomena which are the creation and the propagation of singularities by the interaction of two transversal conormal waves for a semi-linear partial differential system. More precisely, the purpose is to analyze a phenomenon of a loss of piecewise smooth regularity shown by G. Métivier and J. Rauch in [6] and [7].

We consider a first order semi-linear system

$$
L u=f(x, u) .
$$

It is supposed strictly hyperbolic with respect to a timelike function $t$. The coefficients of $L$ are $M \times M$ smooth real matrix. The real function $f$ is smooth. The variable $x$ describes an open neighborhood $\Omega$ of 0 into $\mathbb{R}^{n}$, for which the past is assumed to be a domain of determinacy.

Let $\Sigma_{1}, \Sigma_{1}$ be two characteristic hypersurfaces intersecting transversely along a 2 codimension manifold $\Gamma$.

If $\Sigma$ is a smooth hypersurface, we write $p C_{\Sigma}^{\infty}$ as the space of the piecewise smooth functions with respect to $\Sigma$. Locally, their restriction to each open half-space defined by $\Sigma$ extends in a $C^{\infty}$ function defined on the whole space.

Received by the editors July 22, 1999.

2000 Mathematics Subject Classification. Primary 35L60, 58J47.

Key words and phrases. Microlocal analysis, conormal singularities, semi-linear interaction.

(C)2000 American Mathematical Society 
We work under the following hypotheses:

1. The rays of the bicharacteristics issued from characteristic conormal vectors to $\Gamma$ are transverse to $\Gamma$.

2. The $\mathbb{R}^{M}$ valued function $u$ is a solution of (1.1) in $L_{l o c}^{\infty}$.

3. $u_{\mid t<0} \in p C_{\Sigma_{1}}^{\infty}+p C_{\Sigma_{2}}^{\infty}$.

The hypothesis 1 . implies there is a finite number of characteristic hypersurfaces $\Sigma_{1}, \ldots, \Sigma_{m}(2 \leq m \leq M)$ passing through $\Gamma$. They are two-by-two transverse.

Thanks to J.M. Bony and G. Métivier's works (see [1] (smooth case) and [5] (discontinuous case)), we know that the singularities of $u$ are conormal with respect to $\Sigma_{i}$ for $i=1, \ldots, m$ and $\Gamma$, that is, $W F(u) \subset \bigcup_{i=1, \ldots, m} N\left(\Sigma_{i}\right) \cup N(\Gamma)$ if we write $W F(u)$ as the wave front of $u$ and $N\left(\Sigma_{i}\right)$ (respectively $N(\Gamma)$ ) as the conormal space of $\Sigma_{i}$ (respectively $\Gamma$ ) deprived its vanished section.

More precisely G. Métivier and J. Rauch [7] demonstrated notably that if $\Gamma$ is included in a spacelike hypersurface, then $u$ is piecewise smooth with respect to $\bigcup_{i=1, \ldots, m} \Sigma_{i}$. Note that $m=M$ in this case. Moreover they built an example showing that the hypothesis $m=M$ is essential (see [6]).

In this work we propose a symbolic study of the singularities showing the microlocal mechanism of the creation and the propagation of non-piecewise smooth type singularities. We assume that the number $m$ of characteristic hypersurfaces passing through the locus of interaction is strictly inferior to the size $M$ of the system (1.1). We will see, by a significant example, that the elliptic part of the system originates the apparition of a non-piecewise smooth type singularity on the edge $\Gamma$ which can propagate along the characteristic hypersurfaces $\Sigma_{1}, \ldots, \Sigma_{m}$ if the semi-linearity permits it.

The example we will present is inspired by [6]. Nevertheless our approach is microlocal: we explain the loss of piecewise smooth regularity by a loss of transmission property in the complete symbol of the solution. Note the symbolic forms which appear have generalizations (see [4]) which should allow us to treat more general cases.

We consider the following $5 \times 5$ first order system on $\mathbb{R}^{3}$ :

$$
\left.\begin{array}{r}
\sqrt{2}\left(\partial_{t}+\partial_{x_{1}}\right) u_{1}+\left(\partial_{t}+\partial_{x_{1}}+\partial_{x_{2}}\right) u_{2}=0 \\
\left(\partial_{t}+\partial_{x_{1}}+\partial_{x_{2}}\right) u_{1}+\sqrt{2}\left(\partial_{t}+\partial_{x_{2}}\right) u_{2}=0 \\
\partial_{t} v=u_{1} w_{1} \\
\left(\partial_{t}+2 \partial_{x_{1}}\right) w_{1}+2 \partial_{x_{2}} w_{2}=0 \\
2 \partial_{x_{2}} w_{1}+\left(\partial_{t}-2 \partial_{x_{1}}\right) w_{2}=\psi(t) u_{1} u_{2}
\end{array}\right\}
$$

with $\psi(t)=\mid \begin{gathered}0 \text { if } t<-\varepsilon \\ 1 \text { if } t>-\frac{\varepsilon}{2}\end{gathered}$ for a $\varepsilon>0$.

This system is strictly hyperbolic with respect to the time variable $t$. Writing $\mathbf{H}$ the Heaviside function, we impose the following conditions in the past $\{t<-\varepsilon\}$ :

$$
\begin{aligned}
& \text { for } t<-\varepsilon, \quad u_{1}=\left(t-x_{1}\right)^{k_{1}-1} \mathbf{H}\left(t-x_{1}\right) \text { with } k_{1} \geq 1 \text {, } \\
& u_{2}=\left(t-x_{2}\right)^{k_{2}-1} \mathbf{H}\left(t-x_{2}\right) \text { with } k_{2} \geq 1 \text {, } \\
& v=w_{1}=w_{2}=0 \text {. }
\end{aligned}
$$

In fact, the system (1.2) yields explicitly $u_{j}=\left(t-x_{j}\right)^{k_{j}-1} \mathbf{H}\left(t-x_{j}\right)$ for $j=1,2$. If we consider successively (1.4) as a linear system with respect to $w_{1}, w_{2}$, and (1.3) as a linear equation with respect to $v$, we obtain a global solution $u=\left(u_{1}, u_{2}, v, w_{1}, w_{2}\right)$ which is in $p C_{\Sigma_{1}}^{\infty}+p C_{\Sigma_{2}}^{\infty}$ in the past with $\Sigma_{j}=\left\{t-x_{j}\right\}$ for $j=1,2$. Both 


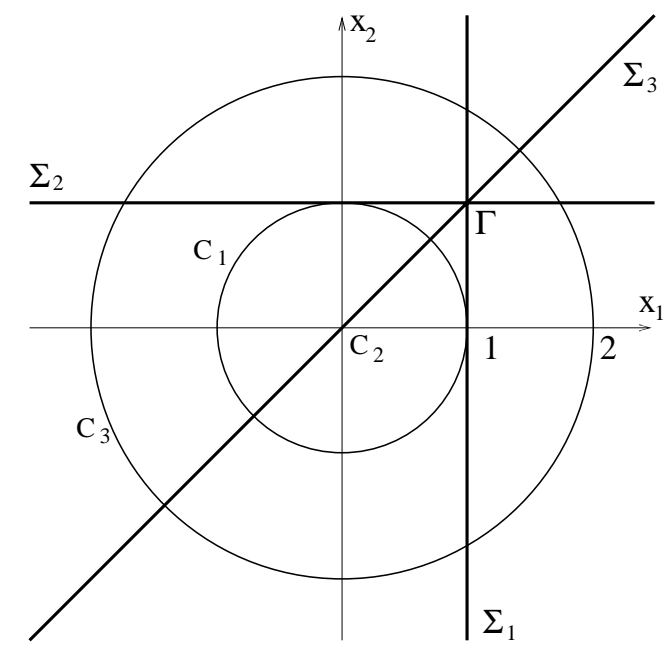

FIGURE 1.

characteristic hypersurfaces $\Sigma_{1}$ and $\Sigma_{2}$ intersect transversely along the edge $\Gamma=$ $\left\{t=x_{1}=x_{2}\right\}$.

Figure 1 shows us that only three characteristic hypersurfaces contain $\Gamma: \Sigma_{1}$, $\Sigma_{2}$ and $\Sigma_{3}=\left\{x_{1}=x_{2}\right\}$. We drew on the plan $\{t=1\}$ the traces of the light cones $C_{1}, C_{2}, C_{3}$ issued from 0 respectively associated with (1.2), (1.3), (1.4).

There is not any characteristic hypersurface for (1.4) passing through the edge $\Gamma$. In other words the system (1.4) is microlocaly elliptic on a neighborhood of $N(\Gamma)$. This partial ellipticity of the system (1.2), (1.3), (1.4) and the "sufficient nonlinearity" of the source term will create non-piecewise smooth type singularities after interaction.

We will prove

Theorem 1.1. On a neighborhood of 0 , near $\Sigma_{3}^{+}=\left\{x_{1}=x_{2}\right\} \cap\left\{t>x_{1}\right\}$, we have $v(x) \equiv c\left(x_{1}-x_{2}\right)^{2 k_{1}+k_{2}-1} \ln \left|x_{2}-x_{1}\right|$ modulo $p C_{\Sigma_{3}}^{\infty} \cap C^{k_{1}+k_{2}-1}$ with $c \neq 0$.

\section{SyMBOLS}

By the change of variables $X_{1}=t-x_{1}, X_{2}=t-x_{2}, X_{3}=x_{1}+x_{2}$, and writing again $x_{1}, x_{2}, x_{3}$ the new variables, we obtain

$$
\left.\begin{array}{r}
\sqrt{2}\left(\partial_{x_{2}}+\partial_{x_{3}}\right) u_{1}+2 \partial_{x_{3}} u_{2}=0 \\
2 \partial_{x_{3}} u_{1}+\sqrt{2}\left(\partial_{x_{1}}+\partial_{x_{3}}\right) u_{2}=0 \\
\left(\partial_{x_{1}}+\partial_{x_{2}}\right) v=u_{1} w_{1} \\
\left(-\partial_{x_{1}}+\partial_{x_{2}}+2 \partial_{x_{3}}\right) w_{1}+2\left(-\partial_{x_{2}}+\partial_{x_{3}}\right) w_{2}=0 \\
2\left(-\partial_{x_{2}}+\partial_{x_{3}}\right) w_{1}+\left(3 \partial_{x_{1}}+\partial_{x_{2}}-2 \partial_{x_{3}}\right) w_{2}=\Psi\left(\frac{1}{2}\left[x_{1}+x_{2}+x_{3}\right]\right) u_{1} u_{2}
\end{array}\right\}
$$

with the new conditions in the past:

$$
\text { for } x_{1}+x_{2}+x_{3}<-2 \varepsilon, \quad \begin{aligned}
& u_{1}=x_{1}^{k_{1}-1} \mathbf{H}\left(x_{1}\right) \text { with } k_{1} \geq 2, \\
& u_{2}=x_{2}^{k_{2}-1} \mathbf{H}\left(x_{2}\right) \text { with } k_{2} \geq 2, \\
& v=w_{1}=w_{2}=0 .
\end{aligned}
$$


Now we have $\Gamma=\left\{x_{1}=x_{2}=0\right\}, \Sigma_{1}=\left\{x_{1}=0\right\}, \Sigma_{2}=\left\{x_{2}=0\right\}, \Sigma_{3}=\left\{x_{1}=\right.$ $\left.x_{2}\right\}$, and for $j=1,2$,

$$
u_{j}=x_{j}^{k_{j}-1} \mathbf{H}\left(x_{j}\right)=U_{j}+g_{j}
$$

with $g_{j} C^{\infty}, U_{j}=\int e^{i x_{j} \xi_{j}} c_{j} \frac{\chi\left(\xi_{j}\right)}{\xi_{j}^{k_{j}}} d \xi_{j}$, and $c_{j}=\frac{1}{2 \pi}(-i)^{k_{j}}\left(k_{j}-1\right)$ !.

Using a microlocal parametrix of (2.3) on a neighborhood of $N(\Gamma)$, and working near 0 so that $\Psi\left(\frac{1}{2}\left[x_{1}+x_{2}+x_{3}\right]\right)=1$, we obtain

$$
w_{1} \equiv P\left(u_{1} u_{2}\right) \text { modulo } C^{\infty}
$$

where $P$ is a pseudo-differential operator of order -1 . Near $N(\Gamma)$ the symbol of $P$ is equal to $\frac{2\left(\xi_{2}-\xi_{3}\right)}{i\left[-\left(\xi_{1}+\xi_{2}\right)^{2}+4\left(\xi_{1}-\xi_{3}\right)^{2}+4\left(\xi_{2}-\xi_{3}\right)^{2}\right]}$.

The microlocal study of the conormal singularities which appear after interaction necessitates the description of diverse class of symbols.

Symbols with one variable of frequency. One recalls that $S^{\mu}\left(\mathbb{R}^{n} \times \mathbb{R}\right)$ is the set of the symbols of order $\mu$ that is the $C^{\infty}$ function $a(x, \xi)$ with $x \in \mathbb{R}^{n}$ and $\xi \in \mathbb{R}$, such that for $\alpha \in \mathbb{N}^{n}, \beta \in \mathbb{N}$, and $\mathbf{K}$ a compact of $\mathbb{R}^{n}$,

$$
\left|\partial_{x}^{\alpha} \partial_{\xi}^{\beta} a(x, \xi)\right| \leq C(1+|\xi|)^{\mu-\beta} \text { for all }(x, \xi) \in \mathbf{K} \times \mathbb{R} .
$$

For a hypersurface $\Sigma$ and $\mu \in \mathbb{R}$, we write $I^{\mu}(\Sigma)$ the space of the distributions $f$ smooth out of $\Sigma$ and which can be written

$$
f(x)=\int e^{i x_{1} \xi_{1}} a\left(x, \xi_{1}\right) d \xi_{1}, \quad a \in S^{\mu}\left(\mathbb{R}^{n} \times \mathbb{R}\right)
$$

near each point of $\Sigma$ in coordinates so that $\Sigma=\left\{x_{1}=0\right\}$. Note the wave front of such distributions is included in $N(\Sigma)$.

With this notion of symbols we can characterize the piecewise smooth regularity by a transmission property of symbols. Indeed we have the well-known following result:

Lemma 2.1. Denoting $x=\left(x_{1}, \ldots, x_{n}\right)=\left(x_{1}, x^{\prime}\right)$ as the variable in $\mathbb{R}^{n}$ and $\Sigma=$ $\left\{x_{1}=0\right\}, p C_{\Sigma}^{\infty}$ is the set of the distributions $f$ of $I^{-1}(\Sigma)$ satisfying (2.7) with

$$
a(x, \xi) \sim \sum_{j \geq 1} \frac{a_{j}\left(x^{\prime}\right)}{\xi_{1}^{j}} \text { for }\left|\xi_{1}\right|>1
$$

In this case $a_{j}\left(x^{\prime}\right)=\frac{1}{2 \pi i^{j}}\left[\partial_{x_{1}}^{j} f\right]_{\Sigma}$ where []$_{\Sigma}$ designates the jump through $\Sigma$.

We write $p C_{k}^{\infty}(\Sigma)=p C_{\Sigma}^{\infty} \cap I^{-k}(\Sigma)$ for $k \in \mathbb{N}^{*}$.

One recalls (see [3]) that the asymptotic expansion (2.8) means that for all $N \in \mathbb{N}^{*}$

$$
a(x, \xi)-\sum_{j=1}^{N-1} a_{j}\left(x^{\prime}\right) \frac{\chi\left(\xi_{1}\right)}{\xi_{1}^{j}} \in I^{-N}(\Sigma)
$$

where $\chi$ is a $C^{\infty}$ function vanishing near 0 so that $\chi\left(\xi_{1}\right)=1$ if $\xi_{1}$ is tall enough. 
Symbols with two variables of frequency. One calls $\tilde{S}^{\mu_{1}, \mu_{2}}$ the set of the symbols with two variables of frequency defined as the $C^{\infty}$ functions $b\left(x, \xi_{1}, \xi_{2}\right)$ with $x \in \mathbb{R}^{n}$ and $\xi_{1}, \xi_{2} \in \mathbb{R}$, so that for $\alpha \in \mathbb{N}^{n}, \beta_{1}, \beta_{2} \in \mathbb{N}$, and $\mathbf{K}$ a compact of $\mathbb{R}^{n}$,

$$
\left|\partial_{x}^{\alpha} \partial_{\xi_{1}}^{\beta_{1}} \partial_{\xi_{2}}^{\beta_{2}} b\left(x, \xi_{1}, \xi_{2}\right)\right| \leq C\left(1+\left|\xi_{1}\right|\right)^{\mu_{1}-\beta_{1}}\left(1+\left|\xi_{2}\right|\right)^{\mu_{2}-\beta_{2}}
$$

for all $\left(x, \xi_{1}, \xi_{2}\right) \in \mathbf{K} \times \mathbb{R}^{2}$.

For $\mu_{1}, \mu_{2} \in \mathbb{R}$, let $\tilde{I}^{\mu_{1}, \mu_{2}}$ be the space of the distributions $f$ on $\mathbb{R}^{n}$ smooth out of $\Sigma_{1}=\left\{x_{1}=0\right\}$ and $\Sigma_{2}=\left\{x_{2}=0\right\}$ such that $f \in I^{\mu_{i}}\left(\Sigma_{i}\right)$ near each point of $\Sigma_{i} \backslash \Gamma$ for $i=1,2$ where $\Gamma=\Sigma_{1} \cap \Sigma_{2}$, and

$$
f(x)=\int e^{i\left(x_{1} \xi_{1}+x_{2} \xi_{2}\right)} b\left(x, \xi_{1}, \xi_{2}\right) d \xi_{1} d \xi_{2}, \quad b \in \tilde{S}^{\mu_{1}, \mu_{2}}
$$

near each point of the edge $\Gamma$. The wave front of such distributions is contained by $N\left(\Sigma_{1}\right) \cup N\left(\Sigma_{2}\right) \cup N(\Gamma)$.

We write $p C_{\Sigma_{1} \cup \Sigma_{1}}^{\infty}$ as the space of the piecewise smooth functions with respect to $\Sigma_{1} \cup \Sigma_{2}$. Locally, their restriction to each quarter space defined by $\Sigma_{1} \cup \Sigma_{2}$ extends in a $C^{\infty}$ function defined on the whole space.

In this way we have a result analogous to Proposition 2.1.

Lemma 2.2. We denote $x=\left(x_{1}, \ldots, x_{n}\right)=\left(x_{1}, x_{2}, x^{\prime \prime}\right)$ as the variable in $\mathbb{R}^{n}$, $\Sigma_{i}=\left\{x_{i}=0\right\}$ for $i=1,2$.

Near each point of the edge $\Gamma=\left\{x_{1}=x_{2}=0\right\}$, the functions of $p C_{\Sigma_{1} \cup \Sigma_{1}}^{\infty}$ are the distributions $f \in \tilde{I}^{-1,-1}$ satisfying (2.9) with

$$
b\left(x, \xi_{1}, \xi_{2}\right) \sim \sum_{j_{1}, j_{2} \geq 1} \frac{b_{j_{1}, j_{2}}\left(x^{\prime \prime}\right)}{\xi_{1}^{j_{1}} \xi_{2}^{j_{2}}} \text { for }\left|\xi_{1}\right|,\left|\xi_{2}\right|>1 .
$$

In this case $b_{j_{1}, j_{2}}\left(x^{\prime \prime}\right)=\frac{1}{4 \pi^{2} i^{j_{1}+j_{2}}}\left[\partial_{x_{1}}^{j_{1}} \partial_{x_{2}}^{j_{2}} f\right]_{\Gamma}$ where []$_{\Gamma}$ designates the jump through $\Gamma$, that is,

$$
[f]_{\Gamma}=f\left(0^{+}, 0^{+}, x^{\prime \prime}\right)-f\left(0^{+}, 0^{-}, x^{\prime \prime}\right)+f\left(0^{-}, 0^{+}, x^{\prime \prime}\right)-f\left(0^{-}, 0^{-}, x^{\prime \prime}\right) .
$$

Let's specify the meaning of the asymptotic expansion (2.10) : for all $P, N \in \mathbb{N}$,

$$
\begin{aligned}
b\left(x, \xi_{1}, \xi_{2}\right)- & \sum_{\substack{1 \leq j_{1} \leq P-1 \\
1 \leq j_{2} \leq N-1}} b_{j_{1}, j_{2}}\left(x^{\prime \prime}\right) \frac{\chi\left(\xi_{1}\right) \chi\left(\xi_{2}\right)}{\xi_{1}^{j_{1}} \xi_{2}^{j_{2}}} \\
& \equiv \sum_{1 \leq j_{1} \leq P-1} b_{j_{1}}^{N}\left(x^{\prime \prime}, \xi_{2}\right) \frac{\chi\left(\xi_{1}\right)}{\xi_{1}^{j_{1}}}+\sum_{1 \leq j_{2} \leq N-1} P b_{j_{2}}\left(x^{\prime \prime}, \xi_{1}\right) \frac{\chi\left(\xi_{2}\right)}{\xi_{1}^{j_{2}}}
\end{aligned}
$$

modulo $\tilde{S}^{-P,-N}$ with $b_{j_{1}}^{N} \in S^{-N}\left(\mathbb{R}^{n-2} \times \mathbb{R}\right)$ and ${ }^{P} b_{j_{2}} \in S^{-P}\left(\mathbb{R}^{n-2} \times \mathbb{R}\right)$.

For $k, k^{\prime} \geq 1$ we say that the symbol $b$ verifying (2.10) is $k, k^{\prime}$-classical if $b_{j_{1}, j_{2}}=0$ for $j_{1} \leq k$ or $j_{2} \leq k^{\prime}$, that is $b \in \tilde{S}^{-k,-k^{\prime}}$.

Let's remark that the asymptotic expansion does not depend on $x_{1}$ in (2.8) and $x_{1}, x_{2}$ in (2.10). In fact we know (see [3]) that every distribution of $I^{\mu}(\Sigma)$ can be written under the form (2.7) with a symbol $a$ not depending on $x_{1}$. We have an analogous result of reduction of amplitude for the elements of $\tilde{I}^{\mu_{1}, \mu_{2}}$ :

Lemma 2.3. Working near a point of the edge $\Gamma$, a distribution $f \in \tilde{S}^{\mu_{1}, \mu_{2}}$, $\mu_{1}, \mu_{2} \in \mathbb{R}$, verifying (2.10) can be written in the form

$$
f(x)=\int e^{i\left(x_{1} \xi_{1}+x_{2} \xi_{2}\right)} c\left(x^{\prime \prime}, \xi_{1}, \xi_{2}\right) d \xi_{1} d \xi_{2}, \quad c \in \tilde{S}^{\mu_{1}, \mu_{2}},
$$


with

$$
c \sim \sum_{\alpha_{1}, \alpha_{2} \in \mathbb{N}} \frac{1}{\alpha_{1} ! \alpha_{1} !} D_{x_{1}}^{\alpha_{1}} D_{x_{2}}^{\alpha_{2}} \partial_{\xi_{1}}^{\alpha_{1}} \partial_{\xi_{2}}^{\alpha_{2}} b_{\mid x_{1}=x_{2}=0}
$$

Here, the asymptotic expansion means that, for all $N \in \mathbb{N}^{*}$,

$$
c-\sum_{\left|\left(\alpha_{1}, \alpha_{2}\right)\right| \leq N-1} \frac{1}{\alpha_{1} ! \alpha_{1} !} D_{x_{1}}^{\alpha_{1}} D_{x_{2}}^{\alpha_{2}} \partial_{\xi_{1}}^{\alpha_{1}} \partial_{\xi_{2}}^{\alpha_{2}} b_{\mid x_{1}=x_{2}=0} \in \sum_{k+k^{\prime}=N} \tilde{S}^{\mu_{1}-k, \mu_{2}-k^{\prime}} .
$$

Action of the pseudo-differential operators. We write $\xi=\left(\xi_{1}, \ldots, \xi_{n}\right)$ as the cotangent variables of $\mathbb{R}^{n}$. We need a new space of symbols : $\bar{S}^{\mu_{1}, \mu_{2}, \nu}=\tilde{S}^{\mu_{1}+\nu, \mu_{2}} \cap$ $\tilde{S}^{\mu_{1}, \mu_{2}+\nu}$ for $\mu_{1}, \mu_{2}, \nu \in \mathbb{R}$, corresponding to a new space of conormal distributions: $\bar{I}^{\mu_{1}, \mu_{2}, \nu}=\tilde{I}^{\mu_{1}+\nu, \mu_{2}} \cap \tilde{I}^{\mu_{1}, \mu_{2}+\nu}$.

Let's describe the action of a pseudo-differential operator on the conormal distributions (see 3] for an idea of the proof).

Lemma 2.4. Let $P$ be a proper pseudo-differential operator of degree $d \leq 0$. Let's write $p(x, \xi)$ as its symbol.

1) If $f \in I^{\mu}(\Sigma), \mu \in \mathbb{R}$, is locally given by (2.7), then Pf $\in I^{\mu+d}(\Sigma)$ can be locally written as

$$
P f=\int e^{i x_{1} \xi_{1}} c\left(x, \xi_{1}\right) d \xi_{1}
$$

with

$$
c\left(x, \xi_{1}\right) \sim \sum_{\alpha} \frac{1}{\alpha !} \partial_{\xi}^{\alpha} p\left(x, \xi_{1}, 0, \ldots, 0\right) D_{x}^{\alpha} a\left(x, \xi_{1}\right) .
$$

2) If $f \in \tilde{I}^{\mu_{1}, \mu_{2}}, \mu_{1}, \mu_{2} \in \mathbb{R}$, is locally given by (2.9), then $P f \in \bar{I}^{\mu_{1}, \mu_{2}, d}$ can be locally written as

$$
P f=\int e^{i\left(x_{1} \xi_{1}+x_{2} \xi_{2}\right)} c\left(x, \xi_{1}, \xi_{2}\right) d \xi_{1} d \xi_{2}
$$

with

$$
\text { with } \quad c\left(x, \xi_{1}, \xi_{2}\right) \sim \sum_{\alpha} \frac{1}{\alpha !} \partial_{\xi}^{\alpha} p\left(x, \xi_{1}, \xi_{2}, 0, \ldots, 0\right) D_{x}^{\alpha} b\left(x, \xi_{1}, \xi_{2}\right) .
$$

As defined in [3], the asymptotic expansion (2.12) means that for all $N \in \mathbb{N}^{*}$,

$$
c\left(x, \xi_{1}\right)-\sum_{|\alpha| \leq N-1} \frac{1}{\alpha !} \partial_{\xi}^{\alpha} p\left(x, \xi_{1}, 0, \ldots, 0\right) D_{x}^{\alpha} a\left(x, \xi_{1}\right) \in I^{\mu+d-N}(\Sigma) .
$$

The meaning of the asymptotic expansion (2.13) is the following : for all $N \in \mathbb{N}^{*}$,

$$
c\left(x, \xi_{1}, \xi_{2}\right)-\sum_{|\alpha| \leq N-1} \frac{1}{\alpha !} \partial_{\xi}^{\alpha} p\left(x, \xi_{1}, \xi_{2}, 0, \ldots, 0\right) D_{x}^{\alpha} b\left(x, \xi_{1}, \xi_{2}\right) \in \bar{I}^{\mu_{1}, \mu_{2}, d-N} .
$$

In that way, if $P$ is a parametrix of a partial differential system elliptic on a neighborhood of $N(\Gamma)$, then its action on the functions of $p C_{\Sigma_{1} \cup \Sigma_{1}}^{\infty}$ yields the apparition of a new type of symbols. These symbols are an asymptotic sum of terms $\sigma_{j_{1}, j_{2}, j}=b_{j_{1}, j_{2}, j}(x) \frac{\rho_{j}\left(x, \xi_{1}, \xi_{2}\right)}{\xi_{1}{ }_{1} \xi_{2}{ }^{j_{2}}}$ where $\rho_{j}$ is a rational function for $\xi_{1}, \xi_{2}$ of degree $-j \leq 0$, without any real pole, and smooth with respect to $x$, and $b_{j_{1}, j_{2}, j}$ is a smooth function. 
More precisely,

Definition 2.5. For $k, k^{\prime}, l \in \mathbb{N}^{*}$, we say that a symbol $b \in \bar{S}^{-k,-k^{\prime},-l}$ is $k, k^{\prime}, l$ prelogarithmic if

$$
b \sim \sum_{\substack{k \leq j_{1} \\ k^{\prime} \leq j_{2} \\ l \leq j}} b_{j_{1}, j_{2}, j}(x) \frac{\rho_{j}\left(x, \xi_{1}, \xi_{2}\right)}{\xi_{1}^{j_{1}} \xi_{2}^{j_{2}}} \text { for }\left|\xi_{1}\right|,\left|\xi_{2}\right|>1
$$

The asymptotic expansion (2.14) means : for all $P, N \in \mathbb{N}^{*}$,

$$
\begin{aligned}
& b\left(x, \xi_{1}, \xi_{2}\right)- \sum_{\substack{k \leq j_{1} \leq P-1 \\
k^{\prime} \leq j_{2} \leq N-1 \\
l \leq j \leq P+N-j_{1}-j_{2}-1}} \rho_{j_{1}, j_{2}, j}\left(x, \xi_{1}, \xi_{2}\right) \frac{\chi\left(\xi_{1}\right) \chi\left(\xi_{2}\right)}{\xi_{1}^{j_{1}} \xi_{2}^{j_{2}}} \\
& \equiv \sum_{\substack{k \leq j_{1} \leq P-1 \\
l \leq j \leq P-j_{1}-1}} b_{j_{1}}^{N}\left(x, \xi_{2}\right) \rho_{j}\left(x, \xi_{1}, \xi_{2}\right) \frac{\chi\left(\xi_{1}\right)}{\xi_{1}^{j_{1}}} \\
&+\sum_{\substack{k^{\prime} \leq j_{2} \leq N-1 \\
l \leq j \leq N-j_{2}-1}} P b_{j_{2}}\left(x, \xi_{1}\right) \rho_{j}\left(x, \xi_{1}, \xi_{2}\right) \frac{\chi\left(\xi_{2}\right)}{\xi_{1}^{j_{2}}}
\end{aligned}
$$

modulo $\tilde{S}^{-P,-N}$ with $b_{j_{1}}^{N} \in S^{-N}\left(\mathbb{R}^{n} \times \mathbb{R}\right)$ and ${ }^{P} b_{j_{2}} \in S^{-P}\left(\mathbb{R}^{n} \times \mathbb{R}\right)$.

If $b$ is such a symbol, the distribution $f$ given by 2.9 is $p C_{\Sigma_{j}}^{\infty}$ near each point of $\Sigma_{j} \backslash \Gamma$ for $j=1,2$. Indeed, near a point of $\Sigma_{1} \backslash \Gamma$ for example, the variable $x_{2}$ does not vanish so we can use the Taylor expansion of $b$ at 0 with respect to $\xi_{2}$ in order to make $f$ under the form (2.7). This Taylor expansion yields a symbol like (2.8).

Nevertheless, $f$ is not $p C_{\Sigma_{1} \cup \Sigma_{1}}^{\infty}$ near the edge $\Gamma$ in general. For example, if $b=$ $\frac{\chi\left(\xi_{1}\right) \chi\left(\xi_{2}\right)}{\xi_{1} \xi_{2}\left(\xi_{1}{ }^{2}+\xi_{2}{ }^{2}\right)}$, then we have $\Delta f \equiv 4 \pi^{2} \mathbf{H}\left(x_{1}\right) \mathbf{H}\left(x_{2}\right)$ modulo $C^{\infty}$, so $\Delta \frac{\partial^{2} f}{\partial x_{1} \partial x_{2}} \equiv 4 \pi^{2} \delta$ and $\frac{\partial^{2} f}{\partial x_{1} \partial x_{2}} \equiv \pi \ln \left(x_{1}^{2}+x_{2}^{2}\right)$ modulo $C^{\infty}$.

Integration transverse to $\Sigma_{1}, \Sigma_{2}, \Gamma$. The following result originates from the properties of integration stated in [2]. Working on a neighborhood of a point on $\Gamma$, we have

Lemma 2.6. Let $f$ be under the form (2.9) with a symbol $b\left(x^{\prime \prime}, \xi_{1}, \xi_{2}\right) \in \tilde{S}^{\mu_{1}, \mu_{2}}$ and $\mu_{1}, \mu_{2}<-1$. If $g$ satisties

$$
\left(\partial_{x_{1}}+\partial_{x_{2}}\right) g=f
$$

and if $g$ is $C^{\infty}$ on a neighborhood of each point of $\Sigma_{3}^{-}=\left\{x_{1}=x_{2}, x_{1}<0\right\}$, then

$$
g(x) \equiv \int e^{i\left(x_{1}-x_{2}\right) \eta} b\left(x^{\prime \prime}, \eta,-\eta\right) d \eta \operatorname{modulo} C^{\infty}
$$

on a neighborhood of each point of $\Sigma_{3}^{+}=\left\{x_{1}=x_{2}, x_{1}>0\right\}$. We note that $b\left(x^{\prime \prime}, \eta,-\eta\right) \in S^{\mu_{1}+\mu_{2}}\left(\mathbb{R}^{n-2} \times \mathbb{R}\right)$.

So if $b$ is $k, k^{\prime}$-classical or $k, k^{\prime}, l$-prelogarithmic, then $g$ defined by (2.16) is respectively $p C_{k+k^{\prime}}^{\infty}\left(\Sigma_{3}\right)$ or $p C_{k+k^{\prime}+l}^{\infty}\left(\Sigma_{3}\right)$ near each point of $\Sigma_{3}^{+}$. 


\section{Applichtion to the proof of Theorem 1.1}

From the expression (2.5) of $u_{1}$ and $u_{2}$, we have $u_{1} u_{2}=U+\tilde{u}_{1}+\tilde{u}_{2}$ with $\tilde{u}_{j} \in p C_{k_{j}}^{\infty}\left(\Sigma_{j}\right)$ for $j=1,2$ and

$$
U(x)=\int e^{i\left(x_{1} \xi_{1}+x_{2} \xi_{2}\right)} c_{1} c_{2} \frac{\chi\left(\xi_{1}\right) \chi\left(\xi_{2}\right)}{\xi_{1}^{k_{1}} \xi_{2}^{k_{2}}} d \xi_{1} d \xi_{2} .
$$

Using the expression (2.6) we obtain $w_{1}=W+P \tilde{u}_{1}+P \tilde{u}_{2}$, where $W=P U$ and $P \tilde{u}_{j} \in p C_{k_{j}+1}^{\infty}\left(\Sigma_{j}\right)$ for $j=1,2$ thanks to Proposition 2.4 i).

So we can write $u_{1} w_{1}=u_{1} W+r_{1}+r_{2}$ with $r_{1}=u_{1} P \tilde{u}_{1} \in p C_{k_{1}}^{\infty}\left(\Sigma_{1}\right)$ and $r_{2}=u_{1} P \tilde{u}_{2} \in p C_{\Sigma_{1} \cup \Sigma_{1}}^{\infty} \cap \tilde{I}^{-k_{1},-k_{2}-1}$.

From equation (2.2) and the condition $v=0$ in the past, we obtain that $v$ is smooth near $\Sigma_{3}^{-}$. So we can study the singularities of $v$ along $\Sigma_{3}^{+}$using Proposition 2.6. The functions $r_{1}, r_{2}$ originate $p C_{k_{1}+k_{2}+1}^{\infty}\left(\Sigma_{3}\right)$ singularities for $v$.

So it remains to study the contribution of the product $u_{1} W$. Proposition 2.4 shows that $W=W_{0}+r$ where $r$ is $C^{\infty}$ and

$$
W_{0}(x)=\int e^{i\left(x_{1} \xi_{1}+x_{2} \xi_{2}\right)} c_{3} \frac{\chi\left(\xi_{1}\right) \chi\left(\xi_{2}\right)}{\xi_{1}^{k_{1}} \xi_{2}^{k_{2}-1}\left(\xi_{1}-\alpha \xi_{2}\right)\left(\xi_{1}-\bar{\alpha} \xi_{2}\right)} d \xi_{1} d \xi_{2}
$$

with $\alpha=\frac{1}{3}+i \frac{2 \sqrt{2}}{2}$ and $c_{3}=\frac{2 c_{1} c_{2}}{3 i}$.

Hence from (2.5) we have $u_{1} W=\left(U_{1}+g_{1}\right)\left(W_{0}+r\right)$. Because $u_{1} r$ is just piecewise smooth with respect to $\Sigma_{1}$, it does not contribute to any singularity of $v$ along $\Sigma_{3}^{+}$. Let's treat the term $g_{1} W_{0}$. The symbol describing its singularities is $k_{1}, k_{2}-1,2$ prelogarithmic. Proposition2.3 allows us to assume that this prelogarithmic symbol does not depend on the variable $x_{3}$. So we can apply Proposition 2.6 to conclude that the contribution of $g_{1} W_{0}$ to calculate $v$ just yields $p C_{k_{1}+k_{2}+1}^{\infty}\left(\Sigma_{3}\right)$ singularities near $\Sigma_{3}^{+}$.

Finally we just need to study the contribution of the term $U_{1} W_{0}$. By convolution in the $\xi_{1}$ variable, the symbol of $U_{1} W_{0}$ is:

$$
\tilde{b}\left(\xi_{1}, \xi_{2}\right)=c_{1} c_{3} \frac{\chi\left(\xi_{1}\right)}{\xi_{1}^{k_{1}}} *_{\xi_{1}} \frac{\chi\left(\xi_{1}\right) \chi\left(\xi_{2}\right)}{\xi_{1}^{k_{1}} \xi_{2}^{k_{2}-1}\left(\xi_{1}-\alpha \xi_{2}\right)\left(\xi_{1}-\bar{\alpha} \xi_{2}\right)} .
$$

We have

Lemma 3.1. Let's write $g(z)=\left[\left(\xi_{1}-z\right)^{k_{1}} z^{k_{1}} \xi_{2}^{k_{2}-1}\left(z-\alpha \xi_{2}\right)\left(z-\bar{\alpha} \xi_{2}\right)\right]^{-1}$.

Then the convolution defined by (3.1) is equivalent to

$$
\sigma\left(\xi_{1}, \xi_{2}\right)=2 i \pi c_{1} c_{3} \mid \begin{aligned}
& \operatorname{Res}\left(g, \alpha \xi_{2}\right) \text { if } \xi_{2}>0 \\
& \operatorname{Res}\left(g, \bar{\alpha} \xi_{2}\right) \text { if } \xi_{2}<0
\end{aligned}
$$

modulo a $k_{1}, k_{2}-1,2$-prelogarithmic symbol plus a $k_{1}, k_{2}+1$-classical symbol.

The notation $\operatorname{Res}\left(g, z_{0}\right)$ designates the residue of $g$ at the pole $z_{0}$.

Let's assume this result provisionally. We obtain:

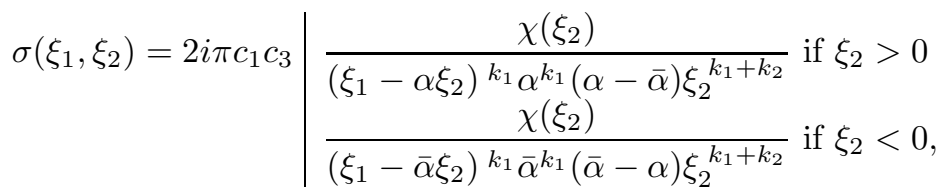


and

$$
\sigma(-\eta, \eta)=2 i \pi c_{1} c_{3} \mid \begin{aligned}
& \frac{\chi(\eta)}{2 i[(-1-\alpha) \alpha]^{k_{1}} \operatorname{Im}(\alpha) \eta^{2 k_{1}+k_{2}}} \text { if } \eta>0 \\
& \frac{\chi(\eta)}{-2 i[(-1-\bar{\alpha}) \bar{\alpha}]^{k_{1}} \operatorname{Im}(\alpha) \eta^{2 k_{1}+k_{2}}} \text { if } \eta<0
\end{aligned}
$$

is a symbol in $S^{-2 k_{1}-k_{2}}(\mathbb{R})$ which does not verify the transmission property characterizing the symbols of the piecewise smooth functions.

Finally Proposition 2.6 implies that near any point of $\Sigma_{3}^{+}$, we can write

$$
v(x) \equiv \int e^{i\left(x_{2}-x_{1}\right) \eta} \sigma(-\eta, \eta) d \eta
$$

modulo a function in $p C_{k_{1}+k_{2}+1}^{\infty}\left(\Sigma_{3}\right)$.

The calculus of Fourier's transformation completes the proof of Theorem 1.1

Remark 3.2. We need the non-linearity to be sufficient if we want the loss of transmission property to occur. For example, if we replace (1.3) by $\partial_{t} v=w_{1}$ the solution is piecewise smooth along $\Sigma_{3}^{+}$and other types of singularities just appear on the edge $\Gamma$.

Remark 3.3. Proposition 3.1 creates a new type of symbol, called "logarithmic". A systematic study of the classical, prelogarithmic and logarithmic symbols ough to allow us to generalize our approach.

Proof of Lemma 3.1. Our aim is to calculate

$$
I_{\mathbb{R}}=\int_{\mathbb{R}} \chi\left(\xi_{1}-z\right) \chi(z) g(z) d z .
$$

Let $\mathbf{K}$ be a compact neighborhood of 0 in $\mathbb{R}$ so that $\chi=1$. At first we study $I_{\mathbf{K}}=\int_{\mathbf{K}} \chi\left(\xi_{1}-z\right) \chi(z) g(z) d z$. The compactness of the domain of integration allows us to expand in power series the functions $z \mapsto\left[\left(z-\alpha \xi_{2}\right)\left(z-\bar{\alpha} \xi_{2}\right)\right]^{-1}$ and $z \mapsto$ $\frac{\chi\left(\xi_{1}-z\right)}{\left(\xi_{1}-z\right)^{k_{1}}}$. We obtain that $I_{\mathbf{K}}$ is a $k_{1}, k_{2}+1$-classical symbol.

Let's treat $I_{\mathbf{K}\left(\xi_{1}\right)}=\int_{\xi_{1}-z \in \mathbf{K}} \chi\left(\xi_{1}-z\right) \chi(z) g(z) d z=\int_{\mathbf{K}} \chi(z) \chi\left(\xi_{1}-z\right) g\left(\xi_{1}-z\right) d z$. We carry out the study of $I_{\mathbf{K}}$ by replacing the first expansion by the expansion of $z \mapsto\left[\left(\xi_{1}-z-\alpha \xi_{2}\right)\left(\xi_{1}-z-\bar{\alpha} \xi_{2}\right)\right]^{-1}$. So $I_{\mathbf{K}\left(\xi_{1}\right)}$ is a $k_{1}, k_{2}-1,2$-prelogarithmic symbol.

Finally we prove that $I_{\mathbb{R}} \equiv \int_{\mathbb{R} \backslash\left(\mathbf{K} \cup \mathbf{K}\left(\xi_{1}\right)\right)} g(z) d z$ modulo the classical and prelogarithmic symbols. The function $g$ is analytic on $\mathbb{C} \backslash\left\{0, \xi_{1}, \alpha \xi_{2}, \bar{\alpha} \xi_{2}\right\}$ and can be integrated in a complex path.

As for $I_{\mathbf{K}}$ and $I_{\mathbf{K}\left(\xi_{1}\right)}, I_{\gamma}=\int_{\gamma} g(z) d z$ is still a $k_{1}, k_{2}+1$-classical symbol and $I_{\gamma\left(\xi_{1}\right)}=\int_{\xi_{1}-z \in \gamma} g(z) d z$ a $k_{1}, k_{2}-1,2$-prelogarithmic one, when $\gamma$ is the half circle defined by Figure 2 .

Accordingly, for a tall enough $R>0, I_{\mathbb{R}}$ is equivalent to $I_{\gamma_{R}}=\int_{\gamma_{R}} g(z) d z$ modulo classical and prelogarithmic symbols, where $\gamma_{R}$ is the closed path defined on the figure.

The Residue Theorem completes the proof. 


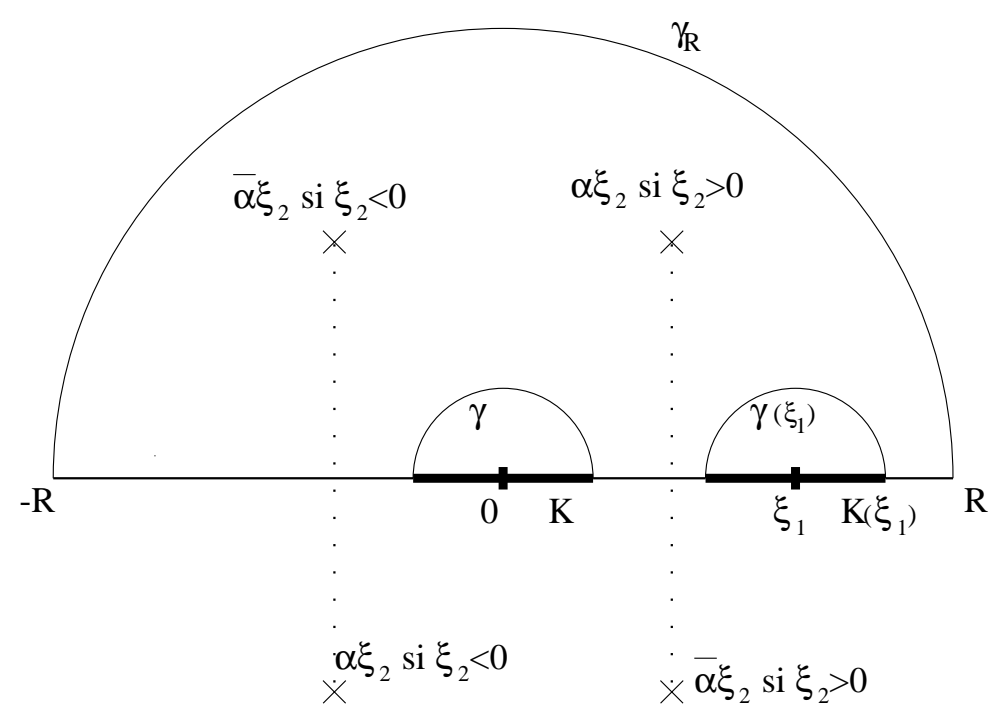

FIGURE 2.

\section{REFERENCES}

1. J.M. Bony. Propagation et interaction des singularités pour des équations aux dérivées partielles non linéaires. In Proc. Int. Cong. Math., 1133-1146, Warszawa, 1983. MR 87f:35156

2. H. Bougrini, A. Piriou, and J.P. Varenne. Propagation et interaction des symboles principaux pour les ondes conormales semi-linéaires. Commun. in Partial Differential Equations, 23:333370, 1998. MR 99c:35003

3. L. Hörmander. The analysis of partial differential operators. Springer-Verlag, New York, 1985.

4. G. Laschon. Symbolic study of the loss of piecewise smooth regularity by interaction of semilinear waves. C. R. Acad. Sci. Paris, 328:865-870, 1999. MR 2000b:35158

5. G. Métivier. Propagation, interaction, and reflection of discontinuous progressing waves. Amer. J. Math., 111:239-289, 1989. MR 90g:35097]

6. G. Métivier and J. Rauch. The interaction of two progressing waves. Springer Lecture Notes in Math., Springer-Verlag, New York, 1402:216-226, 1989. MR 90k:35176

7. G. Métivier and J. Rauch. Interaction of piecewise smooth progressing waves for semilinear hyperbolic equations. Comm. In P.D.E., 15:239-289, 1990. MR 91k:35154

Laboratoire J.A. Dieudonné, Université Nice-Sophia Antipolis, Parc Valrose, F06108 Nice CEDEX 2, France

Current address: Institut de Recherche Mathématique de Rennes, Université Rennes 1, Campus de Beaulieu, F35042 Rennes cedex, France

E-mail address: laschon@maths.univ-rennes1.fr 